\title{
Survival of taylorellae in the environmental amoeba Acanthamoeba castellanii
}

\author{
Julie Allombert ${ }^{1,2,3,4,5}$, Anne Vianney ${ }^{1,2,3,4,5}$, Claire Laugier ${ }^{6}$, Sandrine Petry ${ }^{6^{*}}$ and Laurent Hébert ${ }^{6}$
}

\begin{abstract}
Background: Taylorella equigenitalis is the causative agent of contagious equine metritis, a sexually-transmitted infection of Equidae characterised in infected mares by abundant mucopurulent vaginal discharge and a variable degree of vaginitis, cervicitis or endometritis, usually resulting in temporary infertility. The second species of the Taylorella genus, Taylorella asinigenitalis, is considered non-pathogenic, although mares experimentally infected with this bacterium can develop clinical signs of endometritis. To date, little is understood about the basic molecular virulence and persistence mechanisms employed by the Taylorella species. To clarify these points, we investigated whether the host-pathogen interaction model Acanthamoeba castellanii was a suitable model for studying taylorellae.

Results: We herein demonstrate that both species of the Taylorella genus are internalised by a mechanism involving the phagocytic capacity of the amoeba and are able to survive for at least one week inside the amoeba. During this one-week incubation period, taylorellae concentrations remain strikingly constant and no overt toxicity to amoeba cells was observed.

Conclusions: This study provides the first evidence of the capacity of taylorellae to survive in a natural environment other than the mammalian genital tract, and shows that the alternative infection model, A. castellanii, constitutes a relevant alternative system to assess host-pathogen interactions of taylorellae. The survival of taylorellae inside the potential environmental reservoir A. castellanii brings new insight, fostering a broader understanding of taylorellae biology and its potential natural ecological niche.
\end{abstract}

Keywords: Taylorella equigenitalis, Taylorella asinigenitalis, Contagious equine metritis, Acanthamoeba castellanii, Endosymbiont

\section{Background}

Taylorella equigenitalis is a Gram-negative betaproteobacterium of the Alcaligenaceae family. It is the causative agent of Contagious Equine Metritis (CEM), a World Organisation for Animal Health (OIE), notifiable disease. CEM is a highly contagious sexually-transmitted infection in horses that may lead to abundant mucopurulent vaginal discharge and variable degrees of vaginitis, endometritis and cervicitis [1], causing temporary infertility and occasional abortions have been reported [2]. The presence of T. equigenitalis in stallions does not cause clinical signs and long-term asymptomatic carrier mares have also been reported [3]. These symptomless carrier animals are generally considered to play a key role in the dissemination

\footnotetext{
* Correspondence: sandrine.petry@anses.fr

${ }^{6}$ ANSES, Dozulé Laboratory for Equine Diseases, Bacteriology and

Parasitology Unit, 14430 Goustranville, France

Full list of author information is available at the end of the article
}

of CEM during mating [4]. Unknown prior to its identification in 1977 [5,6], it is generally assumed that the worldwide dissemination of $T$. equigenitalis was the result of the shipment of carrier stallions and mares both within and between countries [2]. As a consequence, many countries implemented strict regulations and disease surveillance, making CEM one of the most regulated equine diseases worldwide [7]. CEM continues to have a major impact on the economy of the equine industry, limiting movement and trade of horses internationally [2].

The second species of taylorellae-Taylorella asinigenitalis-was first reported in 2001 following its isolation from the genital tract of two jacks and a mare $[8,9]$. Although closely related to T. equigenitalis phenotypically [8] and in terms of its genomic characteristics [10], $T$. asinigenitalis has never been reported to cause clinical signs of disease under natural conditions, and is thus considered non-pathogenic. It is important to note that 
despite this apparent lack of pathogenicity, mares experimentally infected with $T$. asinigenitalis can develop clinical signs of metritis and cervicitis [9], and that $T$. asinigenitalis can persist for a long time in donkeys [11]. We therefore consider T. asinigenitalis a potential emerging pathogen that needs to be monitored.

To date, the evolutionary histories of the taylorellae remain unclear. Analysis of the genomes of T. equigenitalis and $T$. asinigenitalis reveals that both species share a very similar gene repertoire $(\approx 85 \%$ of the total genes predicted are common to both Taylorella species) but surprisingly, little DNA sequence identity $[10,12]$. The recently-described taylorellae MultiLocus Sequence Typing (MLST) scheme, which reveals the highly clonal dissemination of taylorellae (especially T. equigenitalis), combined with the emergence of new STs over time suggest that Equidae could be contaminated by an external source of Taylorella originating from an as yet unidentified natural ecological reservoir. Moreover, genome sequence analysis of Alcaligenaceae members suggests that taylorellae diverged by genome reduction from an ancestor which probably had a less specific ecological niche [13] than present day Taylorellae.

Due to the lack of a suitable host model and molecular genetic tools to manipulate taylorellae, the molecular mechanisms involved in the pathogenicity of taylorellae and their host colonisation capacity remain largely unknown. The main information available to date is (i) that T. equigenitalis is able to invade and replicate in equine dermal cells [14] and (ii) that several genes identified in silico as potentially involved in bacterium-host interactions such as genes coding for secretion systems and for proteins containing eukaryotic domains, are present in the taylorellae genomes $[10,12]$.

Since the discovery that Legionella pneumophila can infect and replicate in free-living amoebae [15], there has been an increasing interest in these professional phagocytes which have been used as an alternative host model to study various aspects of host-pathogen interactions and to characterise bacterial virulence mechanisms [16]. Among the bacteria that have evolved to resist destruction by free-living amoebae (hereinafter called ARB for amoeba-resistant bacteria) [16] we can distinguish (i) true symbionts, which cohabit with the amoeba and maintain a stable host-parasite ratio over a specific period and (ii) pathogens able to lyse the amoebae [17]. As a protective environment for ARB, free-living protozoa represent a potential bacterial reservoir and may act as a vector for bacterial dissemination and colonisation of new niches [18].

In this study, we examined the potential of the bactivorous amoeba $A$. castellanii as a host model for $T$. equigenitalis and $T$. asinigenitalis. We assessed (i) the survival capacity of taylorellae in the presence of $A$. castellanii, (ii) the internalisation of taylorellae by $A$. castellanii and (iii) the impact of taylorellae on Acanthamoeba castellanii cultures.

\section{Methods}

\section{Bacterial strains and growth conditions}

The bacterial strains used in this study were as follows: Escherichia coli strain DH5 $\alpha$ (Invitrogen), L. pneumophila serogroup 1 strain Lens [19] and the two recently-sequenced strains T. equigenitalis MCE9 [20] and T. asinigenitalis MCE3 [10]. The axenic A. castellanii strain used in this study was derived from an environmental isolate [21]. Escherichia coli was grown at $37^{\circ} \mathrm{C}$ in Luria-Bertani (LB) medium. Legionella pneumophila was grown at $30^{\circ} \mathrm{C}$ either on buffered charcoal yeast extract (BCYE) agar $\left[10\right.$ g.L $\mathrm{L}^{-1}$ ACES (N-(2-Acetamido)-2-aminoethanesulfonic acid); 10 g.L. $\mathrm{L}^{-1}$ Yeast extract; 2 g.L. $\mathrm{L}^{-1}$ Charcoal; 15 g.L $\mathrm{L}^{-1}$ agar; 0.4 g.L $\mathrm{L}^{-1}$ L-cystein; 0.25 g.L ${ }^{-1}$ $\mathrm{FeNO}_{3} ; \mathrm{pH}$ 6.9] or in BYE liquid medium. Taylorella equigenitalis and T. asinigenitalis were grown at $37^{\circ} \mathrm{C}$ in $5 \%(\mathrm{v} / \mathrm{v}) \mathrm{CO}_{2}$ in air for $48 \mathrm{~h}$ and $72 \mathrm{~h}$ respectively on ready-to-use chocolate agar media (AES Chemunex, Combourg, France). Acanthamoeba castellanii cells were grown at $30^{\circ} \mathrm{C}$ on PYG medium $[0.75 \%(\mathrm{w} / \mathrm{v})$ proteose peptone, $0.75 \%(\mathrm{w} / \mathrm{v})$ yeast extract and $1.5 \%(\mathrm{w} / \mathrm{v})$ glucose] [22] and split once a week.

\section{Bacterial survival following $A$. castellanii co-infection}

Acanthamoeba castellanii cells were infected with E. coli, L. pneumophila, T. equigenitalis or T. asinigenitalis at an MOI (multiplicity of infection) of 50. Infections were synchronised by spinning the bacteria $(880 \times \mathrm{g}, 10 \mathrm{~min})$ and extracellular bacteria removed by washing. Extracellular bacteria were quantified by plating the supernatant, while amoeba-associated bacteria were quantified by plating once the amoebae were lysed (Triton X-100 0.04\%, $30 \mathrm{~min}$ on ice and vigorous pipetting).

\section{Bacteria uptake assay by trypan blue quenching}

Escherichia coli, T. equigenitalis, T. asinigenitalis and $L$. pneumophila phagocytosis by $A$. castellanii was measured by trypan blue quenching as previously described [23]. Briefly, bacterial suspensions of $T$. equigenitalis or $T$. asinigenitalis prepared from plate-grown organisms, together with overnight cultures of E. coli and 3-day cultures of L. pneumophila, were labelled with 5-(and 6-) carboxyfluorescein succinimidyl ester (FSE). Acanthamoeba castellanii monolayers $\left(5 \times 10^{5}\right.$ cells/well $)$ were infected with $2.5 \times 10^{7}$ fluorescent bacteria (MOI 50) for each species. Phagocytosis inhibitors were obtained from SigmaAldrich (St Louis, MO), solubilised in DMSO and used at a concentration of $10 \mu \mathrm{M}$ for Cytochalasin D (CytoD) and $2 \mu \mathrm{M}$ for Wortmannin (Wort). After centrifugation $(880 \times \mathrm{g}, 10 \mathrm{~min})$ to initiate cell-bacterium contact, the plates were incubated at $30^{\circ} \mathrm{C}$ for $30 \mathrm{~min}$. The medium 
was then replaced by $50 \mu$ per well of trypan blue solution to quench the fluorescence of non-internalised bacteria. After 1 min of incubation, the fluorescence of internalised bacteria was measured on an Infinite M200 Pro (Tecan, Männedorf, Germany) at an excitation level of $485 \mathrm{~nm}$ and an emission of $530 \mathrm{~nm}$.

\section{Cytotoxicity to $A$. castellanii}

The number of viable $A$. castellanii cells remaining after infection with E. coli, T. equigenitalis, T. asinigenitalis or L. pneumophila were counted as previously described [21]. Acanthamoeba castellanii monolayers were infected for each bacterium with an MOI of 50. Cell-bacterium contact was initiated by centrifugation $(880 \times g, 10 \mathrm{~min})$ and the plate was incubated at $37^{\circ} \mathrm{C}$ in $5 \%(\mathrm{v} / \mathrm{v}) \mathrm{CO}_{2}$ in air. At indicated time points, the monolayers were washed four times with protease-yeast (PY) extract medium, and then $100 \mu \mathrm{l}$ of PY medium containing 10\% ( $\mathrm{vol} / \mathrm{vol}$ ) of Alamar blue (Invitrogen, Cergy Pontoise, France) was added to tested wells. After a 12-hour incubation, the $\mathrm{OD}_{570}$ and $\mathrm{OD}_{600}$ values were determined. The relative degrees of amoeba mortality were calculated by the following equation: $\left[1\right.$ (mean $\left(\mathrm{OD}_{570}-\mathrm{OD}_{600}\right)_{\text {infected }} /$ mean $\left.\left.\left(\mathrm{OD}_{570}-\mathrm{OD}_{600}\right)_{\text {uninfected }}\right)\right] \times 100$.

\section{Confocal laser scanning observations}

Acanthamoeba castellanii cells were seeded onto sterile glass coverslips in 6 -well plates at $5 \times 10^{6}$ per well in PY medium and allowed to adhere overnight. Monolayers were infected at an MOI of 50 with fluorescein-labelled T. equigenitalis or $T$. asinigenitalis. Infections were synchronised by spinning the bacteria $(880 \times g, 10 \mathrm{~min})$ and extracellular bacteria were removed by washing. Following $4 \mathrm{~h}$ of incubation at $30^{\circ} \mathrm{C}$, cells were fixed with $4 \%$ paraformaldehyde $\left(30 \mathrm{~min}, 4^{\circ} \mathrm{C}\right)$, permeabilised with ice-cold methanol (2 $\mathrm{min})$, washed three times and labelled with rhodamine phalloidin. Coverslips were examined with an inverted confocal microscope (Axiovert $200 \mathrm{M}$; Zeiss, Thornwood, NJ) equipped with a 63X phase-contrast objective lens (Plan Neofluar [Zeiss]; aperture, 1.4, oil).

\section{Light microscope observations of $A$. castellanii infected monolayers}

Acanthamoeba castellanii monolayers were infected at an MOI of 50 with E. coli, L. pneumophila, T. equigenitalis or $T$. asinigenitalis. Cell-bacterium contact was initiated by centrifugation $(880 \times g, 10 \mathrm{~min})$ and incubated at $37^{\circ} \mathrm{C}$ in $5 \%(\mathrm{v} / \mathrm{v}) \mathrm{CO}_{2}$ in air. Monolayers were observed with a Nikon inverted microscope coupled with an Olympus camera (DP120).
Influence of heat-killed $A$. castellanii and $A$. castellanii culture supernatant on taylorellae growth

Microfiltered $(0.22 \mu \mathrm{m})$ supernatant of A. castellanii cultured in PYG medium for 5 days and heat-killed $A$. castellanii cells $\left(100^{\circ} \mathrm{C}, 30 \mathrm{~min}\right)$ were inoculated with a $T$. equigenitalis or $T$. asinigenitalis strain at an $\mathrm{OD}_{600}$ of $0.1,0.2$ and 0.5 . These cultures were incubated for 5 days at $37^{\circ} \mathrm{C}$, either in $5 \%(\mathrm{v} / \mathrm{v}) \mathrm{CO}_{2}$ in air in a static state or aerobically under agitation $(200 \mathrm{rpm})$. Bacterial growth was measured over time by optical density measurement and plate counts.

\section{Results}

Evolution of taylorellae concentrations in co-culture with A. castellanii

To characterise the capacity of $T$. equigenitalis and $T$. asinigenitalis to persist within the free-living amoeba A. castellanii, we performed A. castellanii-taylorellae co-cultures and determined the evolution of extracellular (Figure 1A) and amoeba-associated (Figure 1B) bacterial concentrations over time. Escherichia coli was used as an amoeba-sensitive control bacterium and L. pneumophila, which is able to replicate and evade amoebae, was used as an amoeba-resistant control bacterium. The same evolution of $T$. equigenitalis and $T$. asinigenitalis concentrations was observed over the 7 days of co-culture with A. castellanii: the extracellular taylorellae concentrations decreased about one fold over the experiment period, while the amoeba-associated taylorellae concentrations remained strikingly constant throughout. By comparison, the extracellular and amoeba-associated concentrations of L. pneumophila rapidly rose after two days of incubation and then declined as expected up to and including day 7 , due to the nutrient limitation of the culture medium. As expected, the amount of extracellular and amoebaassociated $E$. coli declined drastically over time during co-culture with $A$. castellanii. These results show that T. equigenitalis and $T$. asinigenitalis persist in association with $A$. castellanii over time.

\section{Acanthamoeba castellanii-associated taylorellae are located intracellularly}

To determine the location of the amoeba-associated taylorellae, confocal laser scanning micrographs of $A$. castellanii cells were performed four hours after infection with fluorescein-labelled $T$. equigenitalis (Figure 2A) or $T$. asinigenitalis (Figure 2B). For both taylorellae, we observed exclusively intracellular bacteria, mainly grouped in clusters. No bacterium was observed attached to the cell surface of the amoeba. Our data show that the persistent amoeba-associated taylorellae are located within the cytoplasm of $A$. castellanii. 


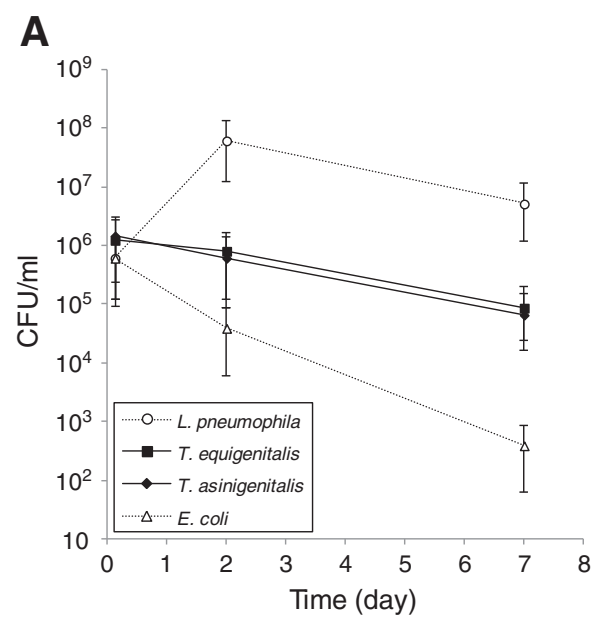

Extracellular bacteria
B

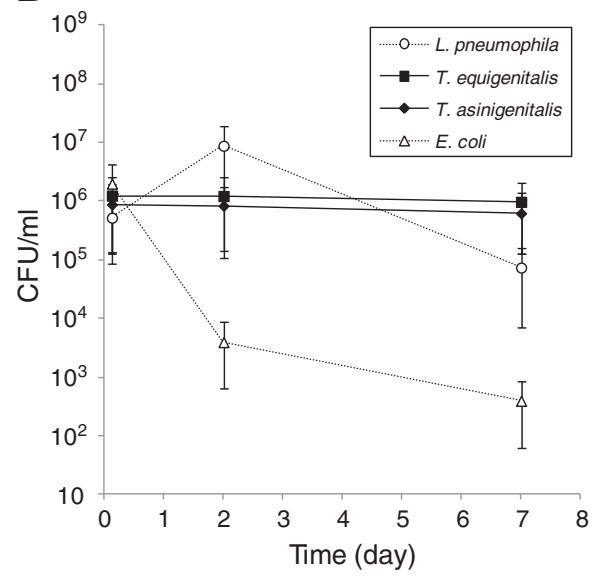

Amoeba-associated bacteria

Figure 1 Taylorella equigenitalis and T. asinigenitalis persist within A. castellanii over time. Evolution of extracellular (A) and amoeba-associated (B) bacterial concentrations following co-cultures with A. castellanii of T. equigenitalis, T. asinigenitalis, E. coli or L. pneumophila. Amoebae were infected at an $\mathrm{MOl}$ of 50 and at indicated time, extracellular and amoeba-associated bacteria following lysis were quantified by plating. The results are expressed in CFU/ml and each bar represents the geometric mean of triplicate wells. The standard deviations are represented by error bars.

Actin polymerisation and phosphoinositide 3-kinase play a key role in taylorellae internalisation

To investigate the uptake mechanism involved in taylorellae internalisation, two chemical inhibitors were used: Cytochalasin D (CytoD), a potent inhibitor of actin polymerisation, and Wortmannin (Wort), an inhibitor of phosphoinositide 3-kinases (PI3K). Bacterial uptake in amoebae was measured by trypan blue quenching of fluoresceinlabelled T. equigenitalis, T. asinigenitalis, E. coli or $L$. pneumophila. Fluorescein-labelled bacteria were used to infect A. castellanii when CytoD or Wort were present, as indicated. After contact, trypan blue was added to quench the fluorescence of non-internalised bacteria and the fluorescence, which was representative of bacterial internalisation by amoebae, was measured (Figure 3). For the four tested bacterial species, amoebae exposed

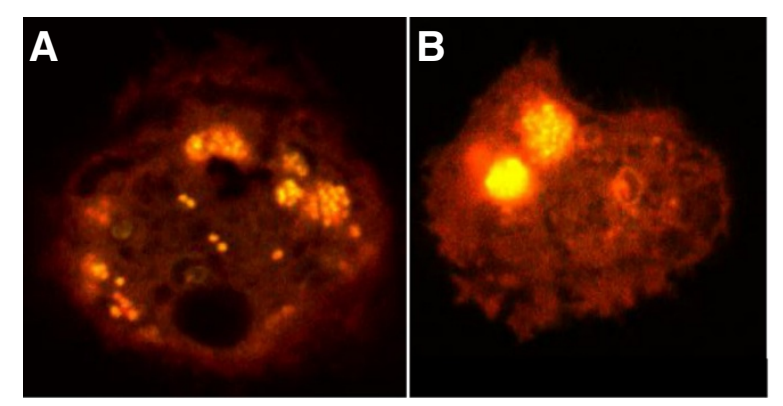

Figure 2 Location of $T$. equigenitalis and $T$. asinigenitalis within A. castellanii. Confocal laser scanning micrographs of $A$. castellanii cells at $4 \mathrm{~h}$ post-infection with fluorescein-labelled $T$. equigenitalis (A) or T. asinigenitalis (B). Similar results were observed in two independent experiments. to CytoD and Wort show a decrease in fluorescence compared to untreated amoebae. The decrease in fluorescence was comparable for all four bacterial species and for both phagocytosis inhibitors. These results suggest that taylorellae are internalised by an uptake mechanism such as phagocytosis, which is dependent upon actin polymerisation and PI3K.

Taylorellae do not obviously alter A. castellanii physiology In order to visualise the impact of taylorellae on $A$. castellanii physiology, we monitored the evolution of $A$. castellanii morphology over a 7-day incubation period in co-culture with T. equigenitalis, T. asinigenitalis, E. coli or L. pneumophila (Figure 4). When A. castellanii was cultivated with the amoeba-sensitive $E$. coli bacteria, we observed that the number of amoebae remained stable and that amoeba cells conserved their typical trophozoite appearance, although they became smaller over time probably as a result of the nutrient limitation of the culture medium. In the presence of the amoeba-resistant L. pneumophila bacteria, we observed a sharp drop in number of amoeba and a drastic change in the surviving $A$. castellanii cell morphology, which gradually shifted to a stress-induced cyst form. The results obtained for co-cultures with taylorellae were similar to those obtained with $E$. coli, with the observation of a conserved trophozoite appearance, a relatively stable concentration of amoeba and a decrease in the size of amoebic cells. There was no evidence of amoebic cyst formation induced by the presence of $T$. equigenitalis or T. asinigenitalis.

To assess the toxicity of bacterial species to $A$. castellanii, amoebae were infected at an MOI of 50 with $T$. 


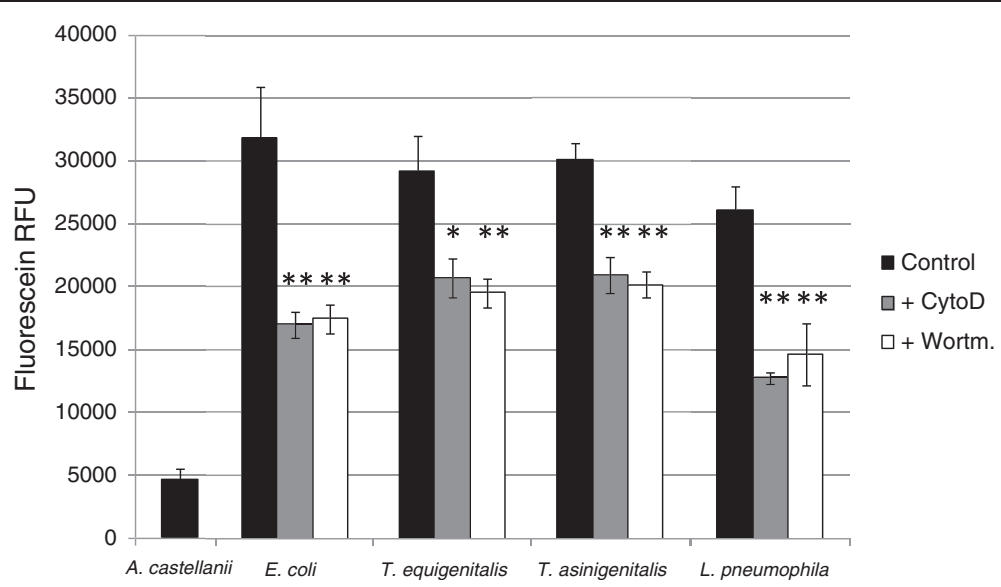

Figure 3 Taylorellae are actively phagocytised by A. castellanii. Bacterial uptake assay by trypan blue quenching. Acanthamoeba castellanii cells were infected with fluorescein-labelled $E$. coli, T. equigenitalis, T. asinigenitalis or L. pneumophila at an $\mathrm{MOI}$ of 50, in the presence, when indicated, of either $10 \mu \mathrm{M}$ of cytochalasin D—an actin polymerization inhibitor (+CytoD)—or $2 \mu \mathrm{M}$ of Wortmanin—a PI3K inhibitor (+Wort). After 30 min of incubation, the medium was replaced by trypan blue solution to quench the fluorescence of non-internalised bacteria. The fluorescence of internalised bacteria was measured using an excitation level of $485 \mathrm{~nm}$ and an emission of $530 \mathrm{~nm}$. Fluorescence data were corrected for differences in labelling efficiency between the tested strains. Each bar represents the mean of triplicate wells and error bars represent the standard deviations. Significant differences from the control, determined by an unpaired tailed Student $t$ test, are indicated by $*(p=0.058)$ and ${ }^{* *}(p<0.05)$.

equigenitalis, T. asinigenitalis, E. coli or L. pneumophila. The viability of amoebic cells in infected monolayers was quantified at indicated time points by using Alamar blue dye (Figure 5). The cytotoxicity of L. pneumophila reached $80 \%$ after one week of incubation, whereas the cytotoxicity of $T$. equigenitalis, $T$. asinigenitalis and $E$. coli to A. castellanii did not exceed $10 \%$ after one week. These data reveal that taylorellae have little cytotoxicity effects on $A$. castellanii.

Taylorellae are not able to grow on dead $A$. castellanii cells To determine the conditions which allowed taylorellae to persist in the presence of amoebae, we measured $T$. equigenitalis and T. asinigenitalis concentrations following $48 \mathrm{~h}$ incubation in the presence of either i) PYG medium, ii) PYG medium in which amoebae were previously cultured for 5 days and iii) heat-killed amoebic cells. No taylorellae growth was observed under any of these conditions (data not shown).

\section{Discussion}

Free-living amoebae are ubiquitous predators that control microbial communities and that have been isolated from various natural sources such as freshwater, soil and air [24]. Following studies on the interaction between ARB pathogens (including Legionella and Chlamydia) and free-

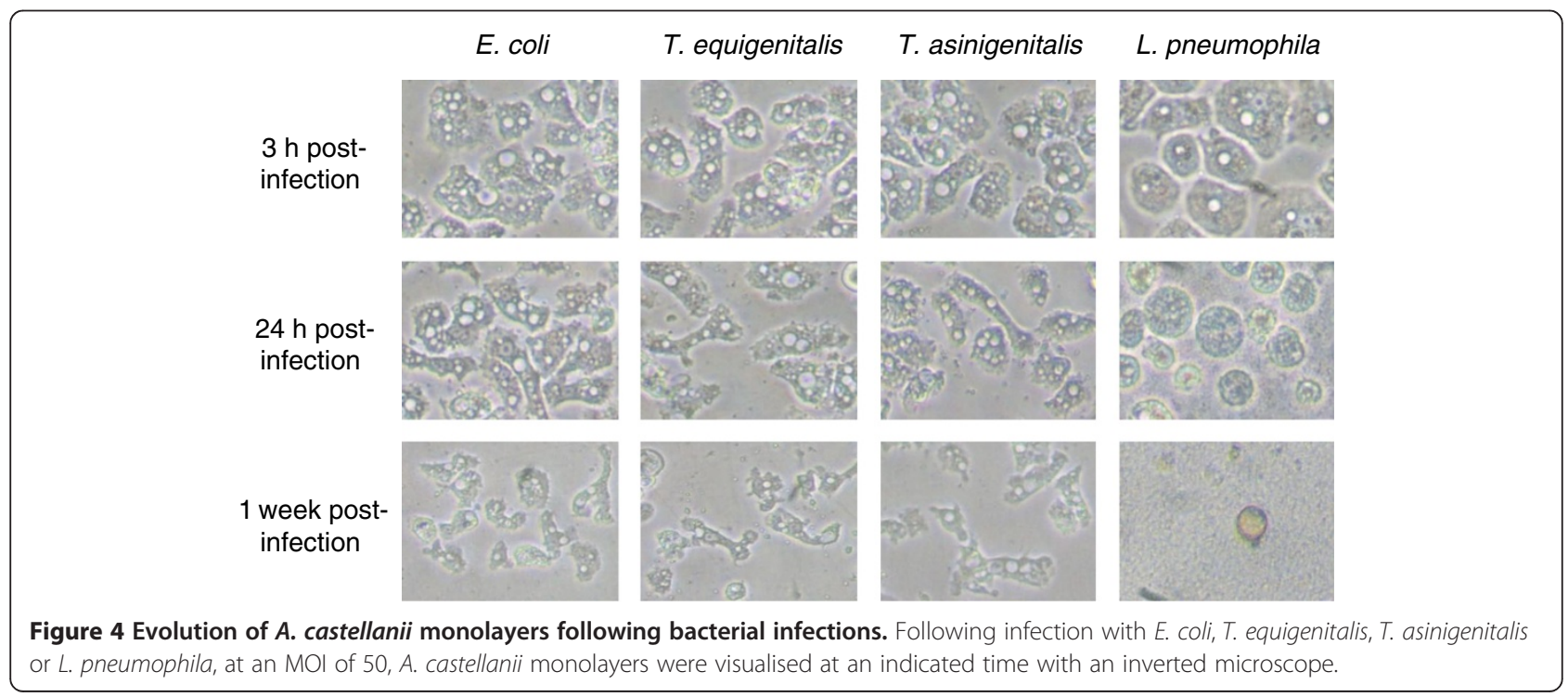




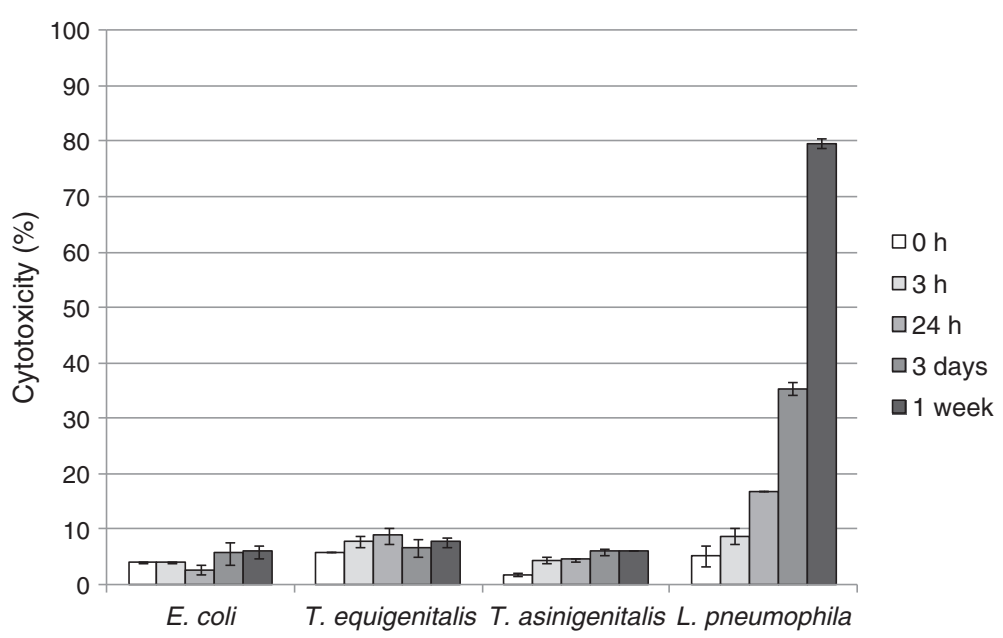

Figure 5 Taylorellae exhibit low cytotoxicity to A. castellanii. Acanthamoeba castellanii were infected with E. coli, T. equigenitalis, T. asinigenitalis or L. pneumophila with an $\mathrm{MOI}$ of 50 . The viability of amoebic cells in infected monolayers was quantified at an indicated time using Alamar blue dye. These data are representative of two independent experiments done in triplicate. Each bar represents the mean of triplicate wells; error bars represent the standard deviations.

living amoebae, it has been suggested that ARB may use free-living amoebae as "training grounds" for the selection of mechanisms of cellular immune evasion [24,25]. In this study, we investigated the interaction of $T$. equigenitalis and $T$. asinigenitalis with the free-living amoeba, $A$. castellanii and showed that taylorellae are able to resist the microbicidal mechanisms of amoebae for a period of at least one week (Figure 1), therefore showing for the first time that taylorellae can be classified as an ARB [16]. However, our results have shown that taylorellae do not induce amoebic death (Figure 4) or cytotoxicity (Figure 5) and indicate that taylorellae are not likely to be considered as amoeba-killing organisms [16].

Confocal microscopic observations of the A castellaniitaylorellae co-cultures also showed that $T$. equigenitalis and $T$. asinigenitalis are found within the cytoplasm of the amoeba (Figure 2), which indicates that taylorellae do not only evade amoebic phagocytosis, but actually persist inside the cytoplasm of this bactivorous amoeba. Moreover, the fact that the phagocytosis inhibitors Wortmannin and Cytochalasin D decrease taylorellae uptake by $A$. castellanii (Figure 3 ) reveals that actin polymerisation and PI3K are involved in taylorellae uptake. This suggests that the internalisation of taylorellae does not result from a specific active mechanism of entry driven by taylorellae, but rather relies on a mechanism involving the phagocytic capacity of the amoeba itself. More investigation on this subject is required to determine the precise effect of taylorellae on organelle trafficking inside the amoeba.

Despite the observed persistence of taylorellae inside amoebae, our results do not allow us to determine whether taylorellae are able to replicate inside an amoeba. During the $7 \mathrm{~d}$ of the A. castellanii-taylorellae co-cultures, we observed a strikingly constant concentration of T. equigenitalis and T. asinigenitalis. This phenomenon may be explained either by the existence of a balance between taylorellae multiplication and the bactericidal effect of the amoeba, or by a concurrent lack of taylorellae multiplication and bactericidal effect of the amoeba. Bacterial clusters observed inside $A$. castellanii could be consistent with taylorellae replication within the amoeba, but given that these photographs were taken only $4 \mathrm{~h}$ after the co-infection, it seems unlikely that the clusters were the result of intra-amoebic multiplication of taylorellae. Moreover, we observed similar fluorescence levels between taylorellae, whereas the progressive halving of fluorescence within daughter cells following each cell division would have generated differing fluorescence levels between bacteria if bacterial replication had occurred. It appears that this clustering phenomenon is more likely due to the presence of aggregated taylorellae prior to entry into A. castellanii or to a trafficking route inside the amoeba that causes gathering of taylorellae at a single location.

In this context, assuming that taylorellae are able to replicate inside amoebae, we can conclude that this phenomenon remains limited and is probably tightly regulated by taylorellae. In order to preserve the protective niche represented by the host cell for as long a duration as possible, it is important that the bacteria do not consume too many nutrients at the detriment of host survival [26]. This statement is consistent with both the limited number of carbon sources which are able to be metabolised by taylorellae [10] and with the absence of observed taylorellae growth in the presence of dead amoebae. Metabolic regulation could be involved in the asymptomatic persistence 
over several years of taylorellae observed in Equidae $[2,27]$, during which taylorellae could be concealed inside host cells as suggested by the observation of equine dermal cells invasion by T. equigenitalis [14]. In this regard, the fact that taylorellae do not induce lysis and that a stable host-parasite ratio remains constant over time, both suggest that taylorellae could be considered a true amoebic endosymbiont, historically defined by Büchner in 1953 as "a regulated, harmonious cohabitation of two nonrelated partners, in which one of them lives in the body of the other" [28].

As highlighted by other intracellular pathogens, protozoan hosts are now considered potential reservoirs and vectors for dissemination of pathogens to mammalian hosts. To date, the natural reservoir of taylorellae is still unknown and it is generally assumed that taylorellae have a limited capacity for survival outside the equine genital tract [29]. In this context, the survival of $T$. equigenitalis and $T$. asinigenitalis in free-living amoebae indicates that protozoa may serve as an environmental reservoir for taylorellae. The fact that this capacity is shared by both species of the Taylorella genus also suggests that this capacity may have been inherited from a common ancestor. It will therefore be important to broaden our comprehension of taylorellae biology to determine the role played by free-living amoebae in the persistence and dispersal of taylorellae in the environment and to determine, for example, if taylorellae could persist within amoebae during encystment and survive exposure to harsh conditions due to the protection afforded by its amoebic host. We believe that the increasing number of metagenome sequence data sets derived from various environments offer promising ways of identifying taylorellae or taylorellae-related bacteria that will contribute to a clearer understanding of taylorellae biology and to novel insights into the evolution of these microorganisms in the near future. Moreover, it appears interesting in this perspective to establish a parallel between taylorellae and the obligate intracellular chlamydiae that were long recognised only as a phylogenetically distinct, small group of closely related microorganisms before the finding that they were symbionts of free-living amoebae and other eukaryotic hosts, leading to a radical change in the perception of chlamydial diversity [30].

Lateral gene transfer (LGT) is considered a key process in the genome evolution of amoebae and amoebaassociated bacteria. The recent analysis of genes predicted to be derived from LGT in the genome of Acanthamoeba sp. [31] showed the presence of 28 genes potentially originating from Betaproteobacteria. Although this analysis did not reveal the presence of genes potentially from taylorellae in Acanthamoeba, these results underline the historical relatedness between free-living amoebae and Betaproteobacteria whose different members have been described as naturally infecting free-living amoebae $[16,32,33]$. On the other hand, no amoeba-related genes were identified during the analysis of taylorellae genomes $[10,12]$. This observation seems coherent with the plausible evolutionary path of taylorellae reported by Gosh et al., [13] which suggests that the evolution of the taylorellae genome is mainly based on a reduction in size, with very few new gene acquisitions since taylorellae's separation from the last Alcaligenaceae common ancestor [13].

The capacity of taylorellae to invade and persist inside amoebae supports the usefulness of this inexpensive and easy-to-manipulate host model to assess various aspects of host-pathogen interactions and to characterise the bacterial persistence mechanisms of taylorellae. However, it should be noted that both T. equigenitalis and T. asinigenitalis behaved in exactly the same way in relation to $A$. castellanii. It is therefore unlikely that all of the variations in virulence level observed in Equidae may be identified. Now that this model has been described, the main limitation to date when studying taylorellae host-pathogen interactions remains the absence of tools needed to genetically manipulate the taylorellae.

\section{Conclusion}

In this study, we investigated the interaction of T. equigenitalis and $T$. asinigenitalis with the free-living amoeba, $A$. castellanii. Taken together, our results show that both taylorellae are able to survive for a period of at least one week in amoebic vacuoles without causing overt toxicity to amoeba cells. The $A$. castellanii-taylorellae co-cultures could therefore be used as a simple and rapid model to assess host-pathogen interactions and to characterise taylorellae bacterial persistence mechanisms. Furthermore, this study provides the first evidence of the capacity of taylorellae to survive in a natural environment other than the mammalian genital tract. A full description of this capacity to interact with another eukaryotic host will undoubtedly contribute to a clearer understanding of taylorellae biology and provide new insight into the evolution of these microorganisms.

\section{Competing interests}

The authors declare that they have no competing interests.

\section{Authors' contributions}

JA Performed and designed the experiments and analyzed the data. JA, AV and LH conceived the study. SP and CL participated in the design of the study and helped to draft the manuscript. LH wrote the paper. All authors read and approved the final manuscript.

\section{Acknowledgements}

Julie Allombert was supported by a PhD fellowship from the French Ministry of Higher Education and Research. This work was supported by grants from the European Regional Development Fund and by the Basse-Normandie Regional Council (http://www.cr-basse-normandie.fr). ANSES's Dozulé Laboratory for Equine Diseases is a member of the Hippolia Foundation. We also wish to thank Delphine Libby-Claybrough, professional translator and native English speaker, for reviewing this article prior to publication. 


\section{Author details}

'International Center for Infectiology Research (CIRI) Legionella pathogenesis group, Université de Lyon, Lyon, France. ${ }^{2}$ Inserm, U1111, Lyon, France. ${ }^{3}$ Ecole Normale Supérieure de Lyon, Lyon, France. ${ }^{4}$ Université Lyon 1, Centre International de Recherche en Infectiologie (CIRI), Lyon, France. ${ }^{5} \mathrm{CNRS}$, UMR5308, Lyon, France. ${ }^{6}$ ANSES, Dozulé Laboratory for Equine Diseases, Bacteriology and Parasitology Unit, 14430 Goustranville, France.

Received: 27 January 2014 Accepted: 13 March 2014

Published: 19 March 2014

\section{References}

1. Wakeley PR, Errington J, Hannon S, Roest HIJ, Carson T, Hunt B, Sawyer J Heath P: Development of a real time PCR for the detection of Taylorella equigenitalis directly from genital swabs and discrimination from Taylorella asinigenitalis. Vet Microbiol 2006, 118(3-4):247-254.

2. Timoney PJ: Horse species symposium: contagious equine metritis: an insidious threat to the horse breeding industry in the United States. J Anim Sci 2011, 89(5):1552-1560.

3. Matsuda M, Moore JE: Recent advances in molecular epidemiology and detection of Taylorella equigenitalis associated with contagious equine metritis (CEM). Vet Microbiol 2003, 97(1-2):111-122.

4. Luddy S, Kutzler MA: Contagious equine metritis within the United States: a review of the 2008 outbreak. J Equine Vet Sci 2010, 30(8):393-400.

5. Crowhurst RC: Genital infection in mares. Vet Rec 1977, 100(22):476.

6. Timoney PJ, Ward J, Kelly P: A contagious genital infection of mares. Vet Rec 1977, 101(5):103.

7. Schulman ML, May CE, Keys B, Guthrie AJ: Contagious equine metritis: artificial reproduction changes the epidemiologic paradigm. Vet Microbiol 2013, 167(1-2):2-8

8. Jang S, Donahue J, Arata A, Goris J, Hansen L, Earley D, Vandamme P, Timoney P, Hirsh D: Taylorella asinigenitalis sp. nov., a bacterium isolated from the genital tract of male donkeys (Equus asinus). Int I Syst Evol Microbiol 2001, 51(3):971-976

9. Katz JB, Evans LE, Hutto DL, Schroeder-Tucker LC, Carew AM, Donahue JM, Hirsh DC: Clinical, bacteriologic, serologic, and pathologic features of infections with atypical Taylorella equigenitalis in mares. J Am Vet Med Assoc 2000, 216(12):1945-1948.

10. Hébert L, Moumen B, Pons N, Duquesne F, Breuil M-F, Goux D, Batto J-M, Laugier C, Renault P, Petry S: Genomic characterization of the Taylorella genus. PLoS One 2012, 7(1):e29953.

11. Donahue JM, Timoney PJ, Carleton CL, Marteniuk JV, Sells SF, Meade BJ: Prevalence and persistence of Taylorella asinigenitalis in male donkeys. Vet Microbiol 2012, 160(3-4):435-442.

12. Hauser $\mathrm{H}$, Richter $\mathrm{DC}$, van Tonder $\mathrm{A}$, Clark L, Preston A: Comparative genomic analyses of the Taylorellae. Vet Microbiol 2012, 159(1-2):195-203.

13. Ghosh W, Alam M, Roy C, Pyne P, George A, Chakraborty R, Majumder S, Agarwal A, Chakraborty S, Majumdar S, Gupta SK: Genome implosion elicits host-confinement in Alcaligenaceae: evidence from the comparative genomics of Tetrathiobacter kashmirensis, a pathogen in the making. PLoS One 2013, 8(5):e64856.

14. Bleumink-Pluym N, ter Laak E, Houwers D, van der Zeijst B: Differences between Taylorella equigenitalis strains in their invasion of and replication in cultured cells. Clin Diagn Lab Immunol 1996, 3(1):47-50.

15. Rowbotham TJ: Preliminary report on the pathogenicity of Legionella pneumophila for freshwater and soil amoebae. J Clin Pathol 1980, 33(12):1179-1183.

16. Greub G, Raoult D: Microorganisms resistant to free-living amoebae. Clin Microbiol Rev 2004, 17(2):413-433.

17. Taylor M, Mediannikov O, Raoult D, Greub G: Endosymbiotic bacteria associated with nematodes, ticks and amoebae. FEMS Immunol Med Microbiol 2012, 64(1):21-31.

18. Snelling WJ, Moore JE, McKenna JP, Lecky DM, Dooley JS: Bacterialprotozoa interactions; an update on the role these phenomena play towards human illness. Microbes Infect 2006, 8(2):578-587.

19. Cazalet C, Rusniok C, Brüggemann H, Zidane N, Magnier A, Ma L, Tichit M, Jarraud S, Bouchier C, Vandenesch F, Kunst F, Etienne J, Glaser P, Buchrieser C: Evidence in the Legionella pneumophila genome for exploitation of host cell functions and high genome plasticity. Nat Genet 2004, 36(11):1165-1173.
20. Hébert L, Moumen B, Duquesne F, Breuil M-F, Laugier C, Batto J-M, Renault P, Petry S: Genome sequence of Taylorella equigenitalis MCE9, the causative agent of contagious equine metritis. J Bacterio/ 2011, 193(7):1785.

21. Hervet E, Charpentier X, Vianney A, Lazzaroni JC, Gilbert C, Atlan D, Doublet P: Protein kinase LegK2 is a type IV secretion system effector involved in endoplasmic reticulum recruitment and intracellular replication of Legionella pneumophila. Infect Immun 2011, 79(5):1936-1950.

22. Khan NA: Pathogenicity, morphology, and differentiation of Acanthamoeba. Curr Microbiol 2001, 43(6):391-395.

23. Charpentier X, Gabay JE, Reyes M, Zhu JW, Weiss A, Shuman HA: Chemical genetics reveals bacterial and host cell functions critical for type IV effector translocation by Legionella pneumophila. PLOS Pathog 2009, 5(7):e1000501.

24. Molmeret M, Horn M, Wagner M, Santic M, Abu Kwaik Y: Amoebae as training grounds for intracellular bacterial pathogens. Appl Environ Microbiol 2005, 71(1):20-28

25. Waterfield NR, Wren BW, Ffrench-Constant RH: Invertebrates as a source of emerging human pathogens. Nat Rev Microbiol 2004, 2(10):833-841.

26. Joseph B, Goebel W: Life of Listeria monocytogenes in the host cells' cytosol. Microbes Infect 2007, 9(10):1188-1195.

27. Breuil MF, Duquesne F, Laugier C, Petry S: Phenotypic and $16 \mathrm{~S}$ ribosomal RNA gene diversity of Taylorella asinigenitalis strains isolated between 1995 and 2008. Vet Microbiol 2011, 148(2-4):260-266.

28. Büchner P: Endosymbiose der Tiere mit Pflanzlichen Mikroorganismen. Basel, Switzerland: Gebundene Ausgabe; 1953

29. Timoney PJ, Harrington A, McArdle J, O'Reilly P: Survival properties of the causal agent of contagious equine metritis 1977. Vet Rec 1978, 102(7):152.

30. Horn M: Chlamydiae as symbionts in eukaryotes. Annu Rev Microbiol 2008, 62(1):113-131

31. Clarke M, Lohan AJ, Liu B, Lagkouvardos I, Roy S, Zafar N, Bertelli C, Schilde C, Kianianmomeni A, Bürglin TR, Frech C, Turcotte B, Kopec KO, Synnott JM, Choo C, Paponov I, Finkler A, Heng Tan CS, Hutchins AP, Weinmeier T, Rattei T, Chu JS, Gimenez G, Irimia M, Rigden DJ, Fitzpatrick DA, Lorenzo-Morales J, Bateman A, Chiu CH, Tang P: Genome of Acanthamoeba castellanii highlights extensive lateral gene transfer and early evolution of tyrosine kinase signaling. Genome Biol 2013, 14(2):R11.

32. Inglis TJ, Rigby P, Robertson TA, Dutton NS, Henderson M, Chang BJ: Interaction between Burkholderia pseudomallei and Acanthamoeba species results in coiling phagocytosis, endamebic bacterial survival, and escape. Infect Immun 2000, 68(3):1681-1686.

33. Marolda CL, Hauröder B, John MA, Michel R, Valvano MA: Intracellular survival and saprophytic growth of isolates from the Burkholderia cepacia complex in free-living amoebae. Microbiology 1999, 145(Pt 7):1509-1517.

doi:10.1186/1471-2180-14-69

Cite this article as: Allombert et al: Survival of taylorellae in the environmental amoeba Acanthamoeba castellanii. BMC Microbiology 2014 14:69.

\section{Submit your next manuscript to BioMed Central and take full advantage of:}

- Convenient online submission

- Thorough peer review

- No space constraints or color figure charges

- Immediate publication on acceptance

- Inclusion in PubMed, CAS, Scopus and Google Scholar

- Research which is freely available for redistribution 